\title{
Handsome cat
}

Cocooned in her blanket

She asks again

If I've seen the kitten's photo

Framed at her bedside

He's a big tabby now

And you should see his markings

You should see him now

$\mathrm{He}$ is such a handsome cat

All alone in a four bed ward

Her youth stolen by tainted blood

Now cancer invades her

All alone, no one visits

Oncology promised to assess her

No notes in the chart

They haven't come

We remind them once again

With each day

Her will drains

She barely eats

And it grows

Twice more

We do our dance

As her walker gathers dust

As hope fades

Have you seen him?

You should see him now

You should see his markings

He's such a handsome cat

\section{Kevin Dueck}

Correspondence to Kevin Dueck, McMaster University Department of Family Medicine, David Braley Health Sciences Centre, Hamilton, Ontario L8P 1H6, Canada; Kevin.Dueck@Medportal.ca

Competing interests None declared

Provenance and peer review Not commissioned; internally peer reviewed.

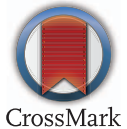

To cite Dueck K. Med Humanit 2017:43:e30.

Published Online First 2 May 2017

Med Humanit 2017;43:e30. doi:10.1136/medhum-2017-011254 